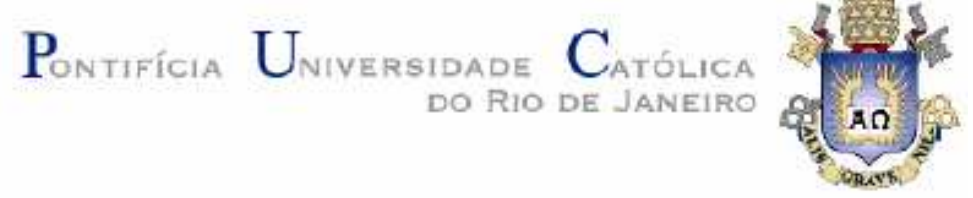

Edson Andrade de Moraes

\title{
Utilização de uma estratégia para identificação de fontes de informação na fase de elicitação
}

Dissertação apresentada como requisito parcial para obtenção do título de Mestre pelo Programa de PósGraduação em Informática da PUC-Rio.

Orientador: Julio Cesar Sampaio do Prado Leite

Rio de Janeiro

Maio de 2008 


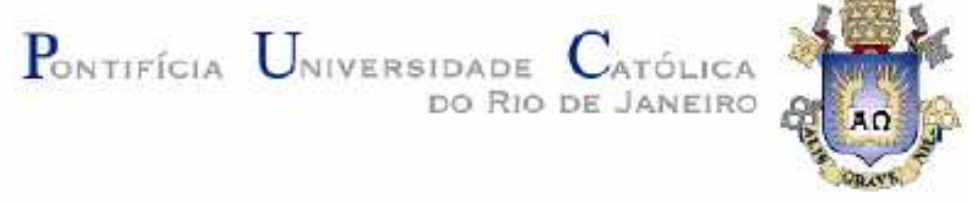

Edson Andrade de Moraes

\title{
Utilização de uma estratégia para identificação de fontes de informação na fase de elicitação
}

\begin{abstract}
Dissertação apresentada como requisito parcial para obtenção do título de Mestre pelo Programa de PósGraduação em Informática da PUC-Rio. Aprovada pela Comissão Examinadora abaixo assinada.
\end{abstract}

\author{
Prof. Julio Cesar Sampaio do Prado Leite \\ Orientador \\ PUC-Rio \\ Prof. Hugo Fuks \\ PUC-Rio \\ Profa. Simone Diniz Junqueira \\ PUC-Rio
}

Prof. José Eugênio Leal

Coordenador(a) Setorial do Centro Técnico Científico - PUC-Rio

Rio de Janeiro, 29 de maio de 2008 
Todos os direitos reservados. É proibida a reprodução total ou parcial do trabalho sem autorização da universidade, do autor e do orientador.

\section{Edson Andrade de Moraes}

Bacharel em Informática e Tecnologia da Informação pela Universidade Estadual do Rio de Janeiro; MBA em Administração e Sistemas de Informação pela Universidade Federal Fluminense.

Ficha Catalográfica

Moraes, Edson Andrade
Utilização de uma estratégia para identificação de
fontes de informação na fase de elicitação / Edson
Andrade de Moraes ; orientador: Julio César Sampaio do
Prado Leite. - 2008.
147 f. ; 30 cm
Dissertação (Mestrado em Informática)-Pontifícia
Universidade Católica do Rio de Janeiro, Rio de Janeiro,
2008. $\quad$ 2. Engenharia de
Inclui bibliografia
1. Informática - Teses.
Requisitos. 3. Elicitação de Requisitos. 4. Universo de
Informações. 5. Fontes de Informação. I. Leite, Julio César
Sampaio do Prado. II. Pontifícia Universidade Católica do
Rio de Janeiro. Departamento de Informática. Ill. Título.

CDD: 004 


\section{Agradecimentos}

A Deus, por me permitir este momento.

A meus Pais, por sempre acreditarem no meu potencial.

A meus gerentes, que sempre apoiaram meus estudos: Tadeu Sousa, Jone Trotte, Antônio Terra, Haroldo Carneiro Leão. 


\section{Resumo}

Moraes, Edson Andrade; Leite, Julio Cesar Sampaio Prado. Utilização de uma estratégia para identificação de fontes de informação na fase de elicitação. Rio de Janeiro, 2008. 147p. Dissertação de Mestrado Departamento de Informática, Pontifícia Universidade Católica do Rio de Janeiro.

Esta dissertação estuda um meio de identificar e selecionar fontes de informação a serem utilizadas na fase de elicitação de requisitos. Utilizamos uma estratégia de identificação e seleção de fontes de informação baseada na modelagem de um Universo de Informações com o uso de uma linguagem de representação gráfica e uma técnica de classificação das fontes de informação que compõem este Universo. Todo o processo é feito com o uso de uma ferramenta de software que dá apoio à execução do método. A ferramenta auxilia no registro das fontes elicitadas e sua consolidação, além de auxiliar na geração de alguns artefatos reduzindo consideravelmente o retrabalho. Um estudo de caso foi efetuado em um problema real de uma empresa de energia, com o objetivo de avaliar os ganhos do uso de uma abordagem estruturada na identificação de fontes de informação ao invés do uso de uma abordagem ad hoc.

\section{Palavras-chave}

Elicitação; fontes de requisitos; fontes de informação; técnicas de elicitação, universo de informações. 


\section{Abstract}

Moraes, Edson Andrade; Leite, Julio Cesar Sampaio Prado. Utilization of an information source identification strategy in the requirements elicitation stage. Rio de Janeiro, 2008. 147p. Msc. Dissertation Departamento de Informática, Pontifícia Universidade Católica do Rio de Janeiro.

This dissertation studies means to identify and select information sources to be used in the requirements elicitation phase. We used an information sources identification and selection strategy based on the modeling of a Universe of Discourse with the use of a graphical representation language and a classification technique of the sources which compose such Universe. The full process is done with the use of a software tool which supports the application of the method. The tool helps in the recording of elicited information sources and its consolidation, besides aiding in the production of some artifacts with a considerable rework reduction. A case study was carried out in a real world problem in an energy company, with the aim of evaluating the gains obtained from the usage of a structured approach for the identification of information sources instead of the use of an ad-hoc approach.

\section{Keywords}

Elicitation; requirements sources; information sources; elicitation techniques, universe of discourse. 


\section{Sumário}

1. Introdução 11

1.1. Descrição Sumária do Problema 12

1.2. Motivação do Trabalho 13

$\begin{array}{ll}\text { 1.3. Estrutura do Trabalho } & 14\end{array}$

2. Elicitação de Requisitos 16

2.1. Engenharia de Requisitos 16

2.2. Elicitação de Requisitos 18

2.3. Universo de Informações e Fontes de Informação 21

2.4. Problemas relacionados às fontes de informação

na literatura de requisitos $\quad 23$

2.5. Técnicas para Seleção e Registro de Fontes de Informação 33

2.6. Comparação das técnicas pesquisadas na literatura. 46

3. Uma estratégia de identificação de fontes de Informação 48

3.1. Visão geral da Estratégia 48

3.2. Identificação e Modelagem das Fontes de Informação 52

3.3. Avaliação das Fontes de Informação 60

4. Ferramenta Universo-I 65

4.1. Requisitos para a construção da ferramenta 66

4.2. Léxico Ampliado da Linguagem 66

4.3. Construção dos Cenários a Partir do Léxico Ampliado 69

4.4. Tecnologias Empregadas $\quad 75$

$\begin{array}{ll}\text { 4.5. Arquitetura } & 77\end{array}$

4.6. Padrões de Projetos Utilizados 77

4.7. Exemplo de Uso da Ferramenta 79

5. Estudo de Caso 85 
5.1. Preparação 85

5.2. Planejamento do Estudo 85

5.3. Estudo de Caso

5.4. Avaliação da Aplicação do Método 97

5.5. Os Formulários de avaliação 97

5.6. Avaliação Quantitativa da Aplicação da Estratégia 104

5.7. Conclusões sobre os Resultados Obtidos 109

6. Conclusão 112

7. Referências Bibliográficas 116 


\section{Lista de figuras}

Figura 1- Classes abstratas de Pontos de Vista

[Kotonya, Sommerville 1996]

Figura 2 - Macroprocesso da Estratégia de Identificação de Fontes de Informação (Leite et al, 20007) 49

Figura 3 - Cinco Passos da Estratégia (Leite et al, 20007). 51

Figura 4 - Detalhamento da atividade AVALIAR (Leite et al, 20007) 60

Figura 5 - Grafo Consolidado anotado com as Estratégias

De Elicitação (Leite et al., 2007) 63

Figura 6 - Matriz de Seleção (Leite et al., 2007). 64

Figura 7 - Grafo do Léxico Ampliado da Linguagem

gerado pela Ferramenta C\&L 69

Figura 8 - Modelo SADT de contrução de Cenários (Leite et al., 2000) 70

Figura 9 - Organização Hierárquica dos Cenários 73

Figura 10 - Grafo dos Cenários Gerados pela Ferramenta C\& L. $\quad 74$

Figura 11 - Organização Temporal dos Cenários $\quad 74$

Figura 12 - Modelo EMF da ferramenta Universo-I 78

Figura 13 - Início da aplicação do método na ferramenta Universo-I $\quad 80$

Figura 14 - Registro da Fonte de Informação $\quad 80$

Figura 15 - Nó de Grafo a ser Utilizado $\quad 81$

Figura 16 - Consolidação dos Grafos de Referência 82

Figura 17 - Grafo Consolidado 82

Figura 18 - Detecção de nomes semelhantes 83

Figura 19 - Suporte ao registro das técnicas de elicitação e atribuição de notas. $\quad 84$

Figura 20 - Grafo obtido da Junção dos Grafos de Referência 93

Figura 21 - Grafo Consolidado Anotado com as

Estratégias de Elicitação 


\section{Lista de tabelas}

Tabela 1 - Propostas para identificação e análise de partes interessadas (Pouloudi, 1999)

Tabela 2 - Lista de Fonte de Informação e

Seu Rastro (Leite et al., 2007) 64

Tabela 3 - Perfil dos participantes do estudo de caso 90

Tabela 4 - Fontes de Informação Identificadas por Participantes 92

Tabela 5 - Fontes de Informação e técnicas de elicitação 93

Tabela 6 - Graus de Relevância e Prioridade atribuídos 96

Tabela 7 - Fontes óbvias e percentuais 105 